\title{
Multicriterion Synthesis of an Electric Circuit for Wireless Power Transfer Systems
}

\begin{abstract}
The paper presents the optimal design of the electric circuit of a Wireless Power Transfer Systems used to recharge the battery of an electric car. A field-circuit model is developed for the purpose of analysis, while a Pareto-like approach - based on SA-MNSGA-III and $\mu$-BiMO, two nature-inspired algorithms - is used for synthesis. An excellent correspondence between the results obtained with the two methods was found. Then, the optimization algorithms could be applied successfully even in more complicated cases, such as WPTSs design.
\end{abstract}

Streszczenie. W artykule zaprezentowano projekt I optymalizację obwodu do bezprzewodowego transferu energii przeznaczonego do ładowania baterii samochodu elektrycznego. Wykorzystano algorytm Pareto. Uzyskano bardzo dobrą zgodność modelu z wynikami eksperymentu. Multikryterialna synteza obwodu do bezprzewodowego transferu energii elektrycznej

Keywords: Wireless Power Transfer Systems, Pareto front, genetic algorithms.

Słowa kluczowe: bezprzewodowy transfer energii, algorytm Pareto.

\section{Introduction}

Wireless Power Transfer Systems (WPTSs) allow charging the onboard batteries of electric vehicles (EVs) without requiring the driver to tinker with the cables and plugs of the conventional battery chargers [1], thus making the use of EVs more user friendly. Moreover, the dynamic version of WPTSs transfers energy onboard a moving vehicle, thus solving two of the main drawbacks of EVs with respect to conventional ones, i.e. their shorter traveling range and longer recharging time.

The physical principle at the basis of WPTSs is the inductive coupling between a transmitting and a receiving coil. The transmitting coil is buried under the road surface and is supplied with high frequency current that generates an oscillating magnetic field. The latter one links the receiving coil, installed beneath the vehicle chassis, and gives rise to an induced voltage that, after being suitably conditioned, charges the EV battery [2].

From the above description, it clearly appears that performance of the WPTSs strongly rely on the synergetic design of the power static converters and the electromagnetic coupling devices. Improving the design of the relevant circuit can be obtained by means of suitable optimization algorithms.

This paper proposes to perform the circuit synthesis by means of algorithms belonging to the class of genetic algorithm starting from a population of $\mathrm{N}$ individuals. In particular, the NSGA-II, SA-MNSGA-III and BiMO are used to solve the synthesis problem [3-6]. Using the optimization algorithms a set of optimal solutions belonging to a Pareto front are obtained. These solutions are compared with the solution corresponding to the parameters of an existing experimental setup, designed using the conventional approach finding that the experimental solutions lays on the same Pareto front, thus validating the proposed design method. Finally, using Finite Element Analysis the robustness of the Pareto front solutions is analyzed considering variations on the axial position of the coils and coil parallelism; the results obtained considering the design parameters relevant to the experimental setup are reported in the paper.

\section{The Problem}

Fig. 1 shows the first-harmonic equivalent (FHE) schema of a prototypal WPTS, designed to charge the battery of an electric mini car and considered as case study. The series compensation topology has been implemented in the receiving side, because of its good efficiency property, and in the transmitting side, because it allows supplying the system with a voltage inverter [7]. The inverter generates a square wave voltage and adjusts its first harmonic amplitude by means of the phase shift technique [2]. Thanks to the filtering effect arising from the $\mathrm{L}-\mathrm{C}$ resonance at the transmitting and the receiving side, the coil currents is and $i_{T}$ are nearly sinusoidal despite the square waveform of the supply voltage vs. For this reason, the equivalent circuits of the WPTSs are usually studied considering only the first harmonics of the electric quantities involved in their functioning and representing them by phasors, as in Fig. 1. The series of the receiving coil and of the resonating capacitor is cascaded by a diode rectifier and a dc-dc converter that charge the battery. These devices are not involved in the processing of reactive power and, in drawing the FHE schema, are represented by the equivalent load resistor $R_{L}$.
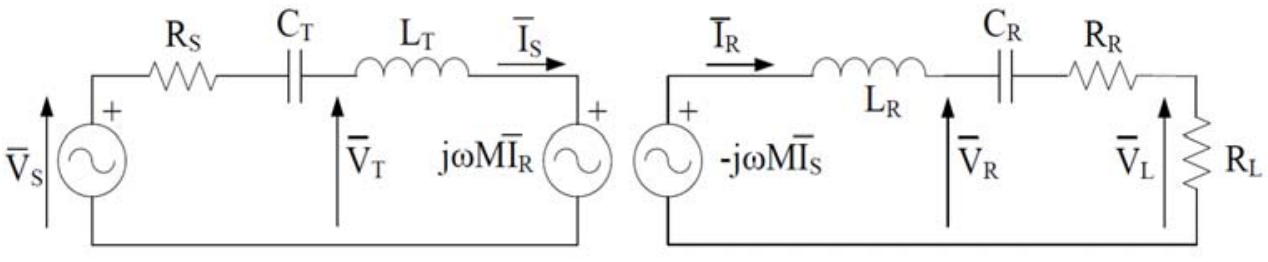

Fig. 1 Electrical FHE schema of the wireless charger system [2] 
The losses in the FHE circuit are mainly given by the parasitic resistances $R_{S}$ and $R_{R}$ of the coil windings. They can be reduced by increasing the section of the wire used to build the coil or reducing the coil currents. This second solution reduces also the losses in the power static converters and hence is preferable. The voltage across the equivalent load $R_{L}$ is determined by the battery voltage and the gain of the dc-dc converter, which cannot be much different from 1 in order to maintain an high power conversion efficiency. The voltage across the $L_{R}-C_{R}$ series is zero because of their resonance and hence the following relation holds for Is.

$$
\bar{V}_{L}=-j \omega M \bar{I}_{S} \Rightarrow \bar{I}_{S}=j \frac{\bar{V}_{L}}{\omega M}
$$

On the other hand, current $I_{R}$ in the receiving coil is determined by the power injected in the battery, so that it can be concluded that the losses in the receiving coil are fixed by the characteristics of the equivalent load, while, by (1), those in the transmitting coil can be reduced if the mutual inductance $M$ is increased.

Considering the $\mathrm{L}_{T}-\mathrm{C}_{T}$ resonance, analysis of the transmitting side of Fig. 1 leads readily to the conclusion that the increase of $M$ requires to increase the supply voltage in the same proportion. On the other hand, supply voltage is somewhat limited by the available grid voltage and cannot be freely increased, so that two opposite requirements about efficiency and supply voltage can be recognized in designing a WPTS.

\section{Inverse problem}

The optimization problem aim is the improvement of the efficiency of the circuit limiting the supply voltage. In this problem three design variables have been selected: 1) the supply frequency $\mathrm{f}$, which varies in the range $[81,90] \mathrm{kHz}$, according to the Society of Automotive Engineers (SAE) specification, 2) the mutual inductance $M$ that varies in the range [10-62] $\mu \mathrm{H}$ in order to comply with the limits on the induced voltage, which in turn depend on the constraints on the supply voltage $V_{S}$ and the coil currents $I_{S}$ and $I_{T} ; 3$ ) the load voltage $\mathrm{V}_{\mathrm{L}}$, applied at the input of the equivalent load and limited in the range [76.4, 127.3] V. Limits in the design variables 1) and 2) come from the pre-design analysis reported in [2] that led to development of the case study WPTS. The design criteria are: f1) the value of the supply voltage $V_{S}$ to be minimized and f2) the power transfer 1efficiency $(1-\eta)$ of the system, to be minimized (i.e. the $\eta$ is maximized):

$$
\begin{gathered}
V_{S}=2 \omega M \frac{P_{B}}{V_{L}} \\
1-\eta=1-\frac{\omega^{2} M^{2} R_{L}}{\left(R_{R}+R_{L}\right)^{2} R_{S}+\omega^{2} M^{2}\left(R_{R}+R_{L}\right)}
\end{gathered}
$$

with $P_{B}$ the power transferred to the battery $\left(P_{B}=560 \mathrm{~W}\right)$ and $V_{S}$ and $V_{L}$ represent the amplitude of the voltages.

\section{Optimization Algorithm}

The optimization algorithms used in the circuit synthesis are all in the class of nature-inspired algorithms. Moreover, both the SA-MNSGA-III and $\mu$-BiMO algorithms are based on the migration concept that allows the modification of the genetic heritage during the optimization process. During the evolution process the SA-MNSGA-III algorithm includes the 'migration' of a new population in the current one, while the $\mu$-BiMO algorithm includes the migration of individuals. The Pareto fronts obtained applying migration algorithms are compared to the ones obtained using the classical NSGA-II algorithm [8].
The Biogeography Based Optimisation (BBO) algorithm was first developed by Simon [9] in 2008 for solving singleobjective optimization problems. In [3,10-12] the multiobjective version of the algorithm was proposed.

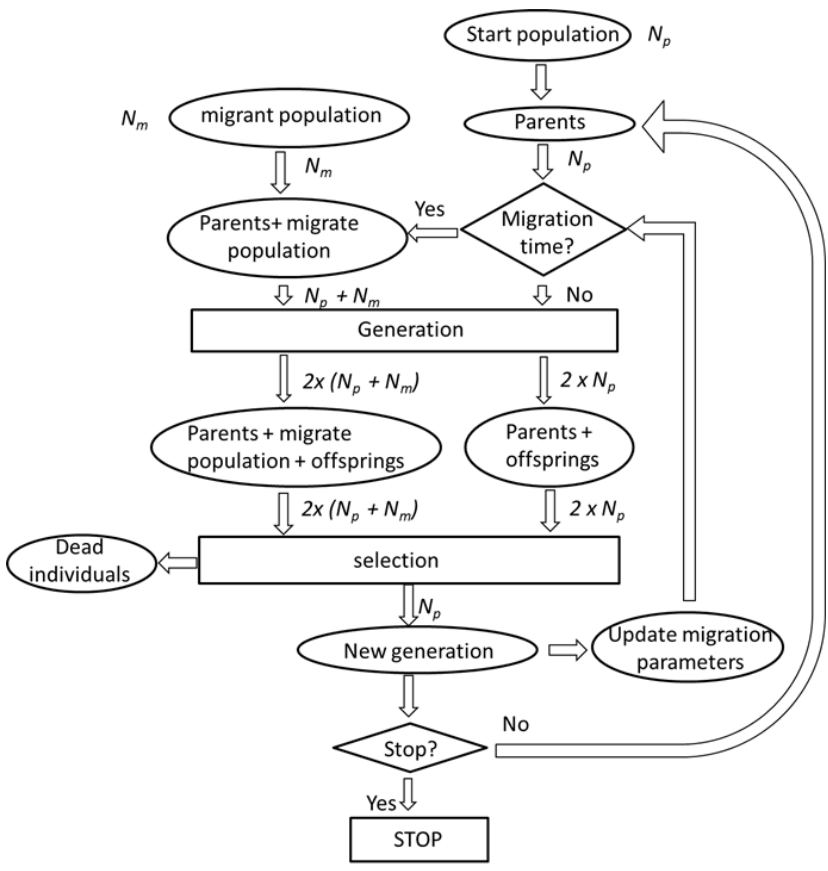

Fig. 2. Schema of the SA-MNSGA-III algorithm

\section{SA-MNSGA-III algorithm}

The Self-Adaptive Migration NSGA (SA-MNSGA) optimization method, described in [6], is based on the classical NSGA-II multi-objective algorithm [8], but was enhanced in order to modify periodically the genetic heritage of the population. In fact, the SA-MNSGA multiobjective optimization method evolves like classical NSGAII algorithm, but includes the periodic 'migration' of new population that modifies the genetic heritage of the current population. It starts from an initial population of $N_{p}$ individual, where usually $N_{p}=20$. The SA-MNSGA version modify NSGA-II in order to allow the migration of a new population of $\mathrm{N}_{\mathrm{m}}$ individuals (with $\mathrm{N}_{\mathrm{m}}<\mathrm{N}_{\mathrm{p}}$ ) inside the current population of $N_{p}$ individuals. The aim of the migration event is the modification of the research space in order to avoid the stop on a local minimum.

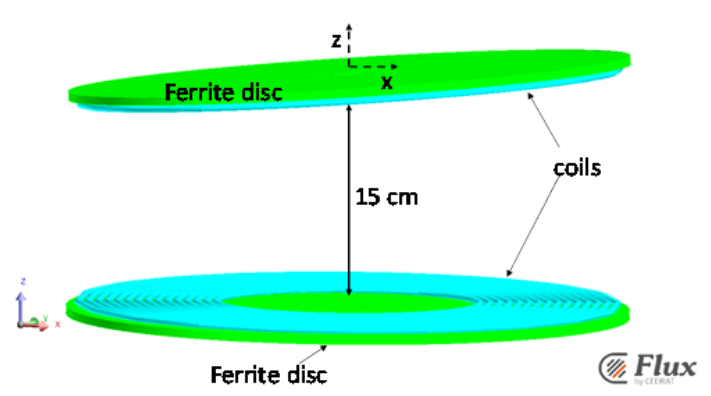

Fig. 3 3D FEM models of the coils.

According to [6] the periodicity of the migration event and the number of immigrated individuals are modified by the algorithm based on the entity of front movement with respect to the previous step, using the Self-Adapting function as in $[5,13,14]$. In practice, if the position of the 
front is changed with respect to the previous step, the migration event is less frequent with respect the case in which the front doesn't move a lot.

With respect to the previous version SA-MNSGA algorithm, in SA-MNSGA-III (Fig. 2), the migration event occurs before the genetic step. In the migration event $\mathrm{N}_{m}$ individuals are generated using the random generation. This way the father selection is made on a larger population that includes both the populations, the current one and the migrated and so formed by $\mathrm{N}_{\mathrm{p}}+\mathrm{N}_{\mathrm{m}}$ individuals instead of $\mathrm{N}_{\mathrm{p}}$ individuals. Then the number of generated individuals is larger with respect to the one in NSGA-II algorithm. Finally, only the best $\mathrm{N}_{\mathrm{p}}$ individual are selected and form the new population.

\section{The $\mu$-BiMO algorithm}

BBO is based on the process of natural immigration and emigration between small islands of species in the search for more friendly habitats, which is observed in nature. Each solution considered is treated as a habitat or island (design vector or individual in genetic algorithms) composed of suitability index variables (SIV, design variables), and each habitat exhibits a quality given by the habitat suitability index (HSI, objective function). The ecosystem, which is the whole set of islands or habitats, is progressively modified by means of two stochastic operators, i.e. migration and mutation: migration improves the $\mathrm{HSI}$ of poor habitats by sharing features from good habitats (exploitation step); in turn, mutation modifies some randomly selected SIV of a few habitats in view of a better search in the design space (exploration step). For each SIV of each habitat a random event $r_{j}$ rules the selection of immigration or emigration operator. If immigration occurs, the current SIV is kept for the next ecosystem, while if emigration occurs the current SIV in the considered habitat (say the i-th habitat) goes extinct and the SIV of another habitat (say the k-th habitat) takes the same SIV position in the i-th habitat of the next ecosystem. The k-th habitat is selected depending on the emigration rate and the SIV.

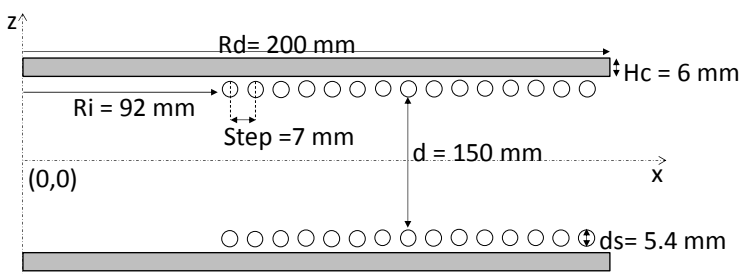

Fig. 4 vertical section of the model with values of geometrical parameters.

The multi-objective version of the algorithm based on the definition of the generalized fitness, which takes into account simultaneously two or more objective functions by exploiting the concept of non-dominated ranking of solutions in the objective space and crowding distance $[3,8,10,11,15]$.

The BiMO algorithm showed good results in different field of applications [16-18]. However when the number of SIV and the number of islands is small, many duplicates of islands occur. To overcome this aspect, the $\mu$-BiMO algorithm was proposed [10]. In this new algorithm, small rocks in the migration of individuals 'help' immigrants to colonize islands that otherwise would not be reached, with the concomitant loss of the individuals who would never reach the ground; then the rocks have the function not to waste habitats that otherwise would never characterize an ecosystem. In particular, during the migration procedure it could happen that good habitats are replaced. To recover this, the discarded habitats are stored in a vector (rock vector) that tracks the habitats. Then, in $\mu$-BiMO instead of generating new habitats randomly, they are taken from the best habitats belonging to the rock vector.

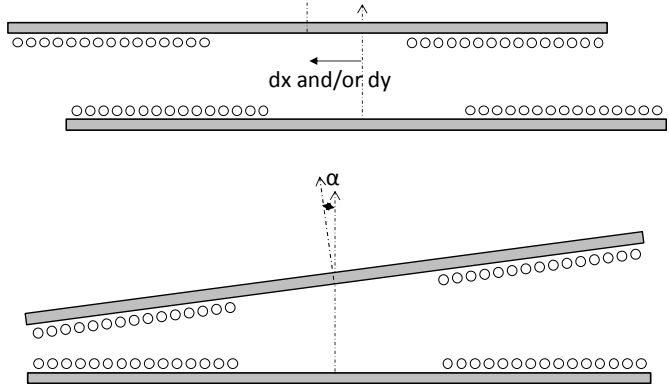

Fig. 5 Schema of coils displacement

\section{FEM model for robustness evaluation}

Fig. 3 shows the 3D model of the experimental device where the two disc ferrite cores, made on 3C95 (Ferroxcube) and the two 15-turns pancake inductors, made on Litz wire, are represented. The sizes of the devices are reported in Fig. 4. The bottom coils was supplied by a current at $85 \mathrm{kHz}$ with rms amplitude equal to $10 \mathrm{~A}$. A time harmonic steady state problem in the magnetic vector potential was solved [19]. The mesh includes 279'000 nodes and 1'597'000 volume elements.

From The FEM model the resulting mutual inductance was evaluated. In order to consider a non-ideal case, two different displacements where applied to the upper coils in order to simulate the misalignment along $\mathrm{x}$ and $\mathrm{y}$ direction, respectively, due to e.g. a bad positioning of the car on the bottom coils. Moreover, the non-parallelism between the two faced coils, due to e.g. mistakes of assembly on the car bottom, was considered. Fig. 5 represents the geometric variations between the two coils considered in the FEM analysis of robustness. In these cases, the mutual inductance was computed using the formula (4) and compared with the one in the ideal case of perfectly facing coils.

\section{Results}

$$
M=\frac{V_{r m s}}{\omega I_{r m s}}
$$

Fig. 6 shows the Pareto fronts obtained using NSGA-II, SAMNSGA-III and $\mu$-BiMO algorithms. The cross points, labelled START in Fig. 6, are initial solutions from which the optimization starts. The circles are the solutions obtained after 26 generations $\left(N_{p}=20\right.$ individuals, 494 calls to objective functions) of NSGA-II. The dots are the solutions obtained after 12 generations $\left(N_{p}=20\right.$ individuals, 455 calls to objective functions) of SA-MNSGA-III. Finally, the triangular points are the solutions obtained after 100 iterations and 500 calls to objective functions of $\mu$-BiMO algorithm (5 islands). Table I reports the values of the design variables at the Pareto front ends obtained with the three optimization algorithms. All the three methods well approximate the Pareto front. In particular, NSGA-II and SA-MNSGA-III found good approximation of the end-points of the front. In turn, point $B$ of $\mu$-BiMO is a dominated solution with respect to the B point found by NSGA-II and B point found by SA-MNSGA-III. This is due to how the boundaries are handled by the methods. In fact, in the 
generation of a new individual, NSGA-II and SA-MNSGA-III consider a value on the border, when a design variable is out of the feasible range, while $\mu$-BiMO re-generate randomly the value. In this problem the end-points of the approximated Pareto front are characterized by values of design variables on the boundaries of the feasible range. Hence, the end-points are well approximated by NSGA-II and SA-MNSGA-III.

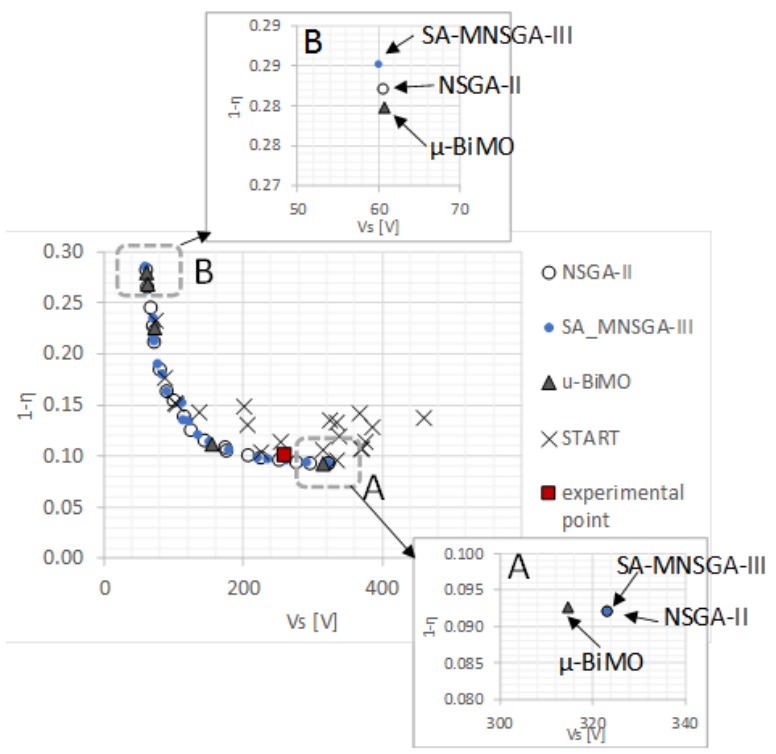

Fig. 6 Pareto front obtained using the three optimization algorithms and experimental point.

Table I. Design variables at the Pareto front extremities

\begin{tabular}{|c|c|c|c|c|c|c|}
\hline algorithm & $f[H z]$ & $\mathrm{V}_{\mathrm{L}}[\mathrm{V}]$ & $\begin{array}{c}\mathrm{M} \\
\text { [uH] }\end{array}$ & $\begin{array}{c}f_{1}(\mathrm{Vs}) \\
{[\mathrm{V}]}\end{array}$ & $\begin{array}{c}f_{2} \\
(1-\eta)\end{array}$ & $\eta$ \\
\hline A-NSGA-II & 90000 & 100.0 & 62.0 & 323.2 & 0.09 & 0.91 \\
\hline B-NSGA-II & 81000 & 97.7 & 10.0 & 60.6 & 0.28 & 0.72 \\
\hline A-SA-MNSGA-III & 90000 & 100.0 & 62.0 & 323.2 & 0.09 & 0.91 \\
\hline B-SA-MNSGA-III & 81252 & 99.5 & 10.0 & 60.1 & 0.29 & 0.71 \\
\hline A- $\mu-B i M O$ & 81916 & 99.5 & 10.1 & 60.7 & 0.28 & 0.72 \\
\hline B- $\mu-B i M O$ & 88247 & 99.5 & 61.3 & 314.6 & 0.09 & 0.91 \\
\hline $\begin{array}{l}\text { Experiment } \\
\text { point }\end{array}$ & 85000 & 82.7 & 31 & 261 & 0.1 & 0.9 \\
\hline
\end{tabular}

The red square point represent the performance achieved by the case study WPTS, whose design variables are reported in the last row of Table I.

Analysis of the Fig. 6 and Table I shows that the design variables obtained at the Pareto front fully comply with the WPTS operation and prove that the two design criteria are conflictual. Moreover, the optimized solutions are in agreement with the design variables selected for the setting up of the prototypal WPTS. In particular, in the figure it clearly appears that operating at higher supply voltages increases the system efficiency because of the reduced losses due to the Is current, while the values reported in the table suggest that a slight increase of $V_{L}$ in the prototype would lead to an increase of the efficiency.

\section{FEM model results}

Table II reports the values of the mutual inductance, M, computed using the equation (4) for the case of the perfectly aligned coils and for the coils misaligned considering $\mathrm{dx}$ equal to $50 \mathrm{~mm}$ or the angle a equal to $5^{\circ}$ as in Fig. 5. The percentage variation with respect the perfectly aligned system is computed as well as the unitary variation. The two misalignments act in an opposite way on the $M$ value.
Table II. Design variables at the Pareto front extremities

\begin{tabular}{ccccc}
\hline $\mathrm{dx}[\mathrm{mm}]$ & $\alpha\left[^{\circ}\right]$ & $\Delta \mathrm{M}[\%]$ & $\begin{array}{c}\mathrm{dM} / \mathrm{dx} \\
{[\mu \mathrm{H} / \mathrm{mm}]}\end{array}$ & $\begin{array}{c}\mathrm{dM} / \mathrm{da} \\
{\left[\mu \mathrm{H} /{ }^{\circ}\right]}\end{array}$ \\
\hline 0 & 0 & -- & -- & -- \\
50 & 0 & -1.99 & -0.0074 & -- \\
0 & 5 & +3.87 & -- & +0.144 \\
\hline
\end{tabular}

\section{Conclusions}

The effectiveness of the optimization method applied to the design of a WPTS has been proved by comparing the design variables obtained at the Pareto front with those of the case study prototype, designed following the traditional trial-and-error method.

The excellent correspondence between the results obtained with the two methods makes it possible to consider that the optimization algorithms presented in this paper could be applied successfully even in more complicated cases, such as WPTSs where the coils have different dimensions [20] or where the compensation networks have a more complex topology [20].

Authors: M. Bertoluzzo, University of Padova, Department of Industrial Engineering, 35131 Padova, Italy, E-mail: manuele.bertoluzzo@unipd.it; P. Di Barba, University of Pavia, Department of Industrial and Information Engineering, Pavia, 27100, Italy, E-mail: paolo.dibarba @unipv.it; F. Dughiero, University of Padova, Department of Industrial Engineering, 35131 Padova, Italy, E-mail: fabrizio.dughiero,@unipd.it; M. E. Mognaschi, University of Pavia, Department of Industrial and Information Engineering, Pavia, 27100, Italy, E-mail: eve.mognaschi@unipv.it; E. Sieni, University of Insubria, via Dunant, 3, 21100 Varese, Italy, E-mail: elisabetta.sieni@uninsubria.it

\section{REFERENCES}

[1] M. Bertoluzzo, N. Zabihi, G. Buja, Overview on battery chargers for plug-in electric vehicles, in: 2012 15th International Power Electronics and Motion Control Conference (EPE/PEMC), 2012: p. https://doi.org/10.1109/EPEPEMC.2012.6397461.

[2] G. Buja, M. Bertoluzzo, K. N. Mude, Design and Experimentation of WPT Charger for Electric City Car, IEEE Transactions on Industrial Electronics. 62 (2015) 7436-7447. https://doi.org/10.1109/TIE.2015.2455524.

[3] P. Di Barba, M.E. Mognaschi, A. Savini, S. Wiak, Island biogeography as a paradigm for MEMS optimal design, International Journal of Applied Electromagnetics and Mechanics. 51 (2016) S97-S105. https://doi.org/10.3233/JAE2015.

[4] P. Di Barba, F. Dughiero, M. Forzan, E. Sieni, Handling sensitivity in multiobjective design optimization of MFH inductors, IEEE Transactions on Magnetics. (2017) 1-1. https://doi.org/10.1109/TMAG.2017.2658728.

[5] E. Sieni, P. Di Barba, M. Forzan, Migration NSGA: method to improve a non-elitist searching of Pareto front, with application in magnetics, Inverse Problems in Science and Engineering. 24 (2016) https://doi.org/10.1080/17415977.2015.1047366.

543-566.

[6] E. Sieni, P. Di Barba, F. Dughiero, M. Forzan, Self-adaptive migration NSGA and optimal design of inductors for magnetofluid hyperthermia, Engineering Computations. 35 (2018) 1727-1746. https://doi.org/10.1108/EC-05-2016-0186.

[7] R. K. Jha, G. Buja, M. Bertoluzzo, S. Giacomuzzi, K. N. Mude, Performance Comparison of the One-Element Resonant EV Wireless Battery Chargers, IEEE Transactions on Industry Applications. $\quad 54 \quad$ (2018) 2471-2482. https://doi.org/10.1109/TIA.2018.2796058.

[8] K. Deb, A. Pratap, S. Agarwal, T. Meyarivan, A fast and elitist multiobjective genetic algorithm: NSGA-II,

Evolutionary Computation, IEEE Transactions on DOI 10.1109/4235.996017. 6 (2002) 182-197.

[9] D. Simon, Biogeography-Based Optimization, Evolutionary Computation, IEEE Transactions On. 12 (2008) 702-713. https://doi.org/10.1109/TEVC.2008.919004.

[10]M.E. Mognaschi, Micro biogeography-inspired multi-objective optimisation for industrial electromagnetic design, Electronics 
Letters. 53 https://doi.org/10.1049/el.2017.3072.

[11]P. Di Barba, F. Dughiero, M.E. Mognaschi, A. Savini, S. Wiak Biogeography-Inspired Multiobjective Optimization and MEMS Design, IEEE Transactions on Magnetics. 52 (2016) 1-4. https://doi.org/10.1109/TMAG.2015.2488982.

[12]P. Di Barba, L. Fassina, G. Magenes, M.E. Mognaschi, Automated optimal design of wells for electromagnetic cell stimulation, ELECTROTECHNICAL REVIEW. 1 (2019) 3-6. https://doi.org/10.15199/48.2019.05.01.

[13]P. Di Barba, F. Dughiero, M. Forzan, E. Sieni, Self-adaptive NGSA algorithm and optimal design of inductors for magnetofluid hyperthermia, COMPEL - The International Journal for Computation and Mathematics in Electrical and Electronic Engineering. 36 (2017) 535-545.

[14]P. Di Barba, F. Dughiero, M. Forzan, E. Sieni, Migrationcorrected NSGA-II for improving multiobjective design optimization in electromagnetics, International Journal of Applied Electromagnetics and Mechanics. 51 (2016) 161-172. https://doi.org/10.3233/JAE-150171.

[15]K. Deb, Multi-objective optimization using evolutionary algorithms, 1st ed, John Wiley \& Sons, Chichester ; New York, 2001.

[16]P. Di Barba, M.E. Mognaschi, A. Krawczyk, The biogeographyinspired optimization for the design of coils for nerve stimulation, in: IEEE, 2017: pp. 542-545. https://doi.org/10.1109/EUROCON.2017.8011170.

[17]P. Di Barba, M.E. Mognaschi, P. Venini, S. Wiak, Biogeography-inspired multiobjective optimization for helping MEMS synthesis, Archives of Electrical Engineering. 66 (2017). https://doi.org/10.1515/aee-2017-0046.

[18]Di Barba P., F. Dughiero, M. Forzan, M.E. Mognaschi, Sieni E., New solutions to a multi-objective benchmark problem of induction heating: an application of computational biogeography and evolutionary algorithms, Archives of

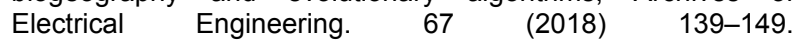
https://doi.org/10.24425/118997.

[19]FLUX, (Altair): https://altairhyperworks.com/product/flux, (n.d.).

[20]M. Bertoluzzo, G. Buja, H. K. Dashora, Design of DWC System Track with Unequal DD Coil Set, IEEE Transactions on Transportation Electrification. 3 (2017) 380-391. https://doi.org/10.1109/TTE.2016.2646740. 